\title{
Análisis Teórico y Experimental de la Potencia, Eficiencia Térmica y Emisiones de Cocinas Industriales que usan Gas Licuado de Petróleo
}

\author{
Freddy J. Rojas*, Fernando O. Jiménez y Jasmine Soto \\ Facultad de Ingeniería, Sec. Ing. Mecánica, Pontificia Universidad Católica del Perú, Av. Universitaria 1801, \\ San Miguel, Lima-Perú (e-mail: fjrojas@pucp.edu.pe; ojimene@pucp.edu.pe; jasmine.soto@pucp.pe)
}

* autor a quien debe ser dirigida la correspondencia.

Recibido Ago. 13, 2018; Aceptado Nov. 5, 2018; Versión final Dic. 18, 2018, Publicado Ago. 2019

\begin{abstract}
Resumen
El objetivo del trabajo que se presenta es mejorar el rendimiento térmico de cocinas industriales que utilizan gas licuado de petróleo (GLP) a fin de promover el buen uso del recurso en la ciudad de Lima. Los experimentos fueron realizados en un banco de ensayos, donde se registraron variables físicas que utiliza el modelo del prototipo. Luego se determinó la potencia y rendimiento de varias cocinas industriales del mercado peruano para comparar con el diseño de la cocina prototipo considerando dos normas internacionales. El quemador dio un amplio rango de potencia según la presión de trabajo utilizado y un alto rendimiento de 59\% comparado con las cocinas que se comercializan a nivel nacional que en promedio están en $45 \%$. La ventaja de esta cocina prototipo es que usa presiones de operación entre 28 a 60 mbar comparada con las cocinas del mercado peruano que usan presiones entre 3 a 6 bar, disminuyendo de esta manera los riesgos asociados a incendio y explosión.
\end{abstract}

Palabras clave: cocinas industriales, eficiencia térmica, gas licuado de petróleo.

\section{Theoretical and Experimental Analysis of the Power, Thermal Efficiency and Emissions of Industrial Stoves using Liquefied Petroleum Gas}

\begin{abstract}
The objective of the work presented in this paper is to improve the thermal performance of industrial stoves that use liquefied petroleum gas (LPG) in order to promote good use of the resource in the city of Lima. The tests were carried out in a test bench, where physical variables were registered to be used in the prototype model. Then, the power and performance of several industrial stoves in the Peruvian market were determined to compare with the design of the prototype device considering two international standards. The burner gave a wide range of power according to the working pressure used and a high efficiency of $59 \%$ compared to the stoves that are nationwide sold, which are on average $45 \%$. The advantage of this prototype device is that it uses operating pressures between 28 to 60 mbar compared to kitchens in the Peruvian market that use pressures between 3 to 6 bar, thus reducing the risks associated with fire and explosion.
\end{abstract}

Keywords: industrial stoves, thermal efficiency, liquefied petroleum gas. 


\section{INTRODUCCIÓN}

Según estudios realizados por la Agencia Internacional de la Energía (2014) el consumo de combustibles fósiles se ha ido incrementando hasta alcanzar casi el $80 \%$ de la energía consumida en el Perú. Esto trae como resultado un impacto ambiental en la calidad del aire y como consecuencia un problema en la salud de los pobladores (SENAMHI, 2014). Actualmente los vendedores de cocinas industriales en el Perú ofrecen distintos tipos de cocinas con diferentes tipos de quemadores, sin embargo, no especifican la potencia entregada por las cocinas. En la realidad los usuarios de estas cocinas en los comercios como los restaurantes lo usan con una presión de operación por encima de los 3 bar es decir a altas presiones sin interesar la eficiencia y las emisiones que se dan lugar, debido a que en el Perú no hay norma que lo regule. Pruebas preliminares a estas cocinas industriales en el mercado peruano mencionan la baja eficiencia térmica, es decir de $40 \%$ o menor eficiencia, y esta no está indicada en sus datos técnicos, esto se ve reflejado en el color amarillo de la llama por la mala combustión y como resultado adicional consume más combustible del que debería (Ramos, 2016).

Investigadores han buscado mejorar la eficiencia de las cocinas industriales, como resultado de sus investigaciones han descubierto que existen diversos factores que pueden afectar la combustión de la mezcla aire-combustible. En un trabajo de investigación en Tailandia se investigó que la cantidad de puertos en el quemador y el ángulo de salida de estos, son los parámetros influyentes en el funcionamiento del quemador (Aroonjattham y Suttikiat, 2015). En la India un trabajo de investigación reveló que las modificaciones en las características geométricas de la boquilla del inyector puede mejorar la eficiencia de la cocina y reducir la cantidad de hollín (Basu et al., 2008). En otro trabajo de investigación en la India se determinó que existen otros parámetros que influyen en la eficiencia, como son el diámetro del recipiente y la distancia que existe entre el recipiente y el quemador (Agarwal et al., 2015). En Colombia se realizó una investigación sobre los quemadores que usan gas natural, donde se concluyó que el exceso de aire tiene una influencia negativa en la temperatura de los productos, y que el precalentamiento del aire tiene una influencia positiva en la temperatura (Serrano y Carranza, 2005).

Otra investigación en la India usó gas licuado de petróleo (GLP) en cocinas domésticas, determinaron que la eficiencia se afecta al considerar varios parámetros de diseño en el quemador (Surange et al., 2014). En México se investigó que las secciones convergentes en las cámara de mezclado tienen mejor arrastre y por lo tanto repercute en la eficiencia, se usa para esto el código numérico PHOENICS (Del et al., 2009). En el Perú, los autores también han evaluado eficiencia térmica en cocinas domésticas, concluyendo en una mejora considerando diámetro del inyector, diseño del mezclador y posición de la olla con respecto al quemador usando GLP (Rojas et al., 2017); y usando gas natural en altitud (Rojas et al., 2017). En Colombia se realizó un estudio teórico- práctico sobre el diseño y comportamiento de los quemadores de premezcla con efecto inductor, haciendo un énfasis en los quemadores de premezcla con aire inductor (Agudelo y Salinas, 2010). En Brasil, se analizó numéricamente el proceso de combustión, en un quemador tipo corona de operación atmosférica, se analizó la configuración geométrica del quemador, caracterizando la respuesta fluidodinámica que genera, utilizando un mecanismo cinético global (Cancino et al., 2007). En un estudio de investigación se informa sobre el rendimiento en términos de distribuciones de temperatura axial y radial, eficiencia térmica y emisiones de $\mathrm{CO}$ y NO de una estufa de cocina de gas licuado de petróleo a mediana escala con un quemador poroso de dos capas; con una potencia de quemador en el rango de 5-10 kW, se investigan los efectos de la relación de equivalencia en las distribuciones de temperatura radial y axial, la eficiencia térmica y las emisiones. (Mishra et al., 2015).

Como solución se propone variar la cantidad de puertos del quemador, la geometría del mezclador y el diámetro del inyector, de modo de lograr una potencia mínima de $3.75 \mathrm{~kW}$. En el presente documento se tiene en cuenta los conceptos de termodinámica y mecánica de fluidos para obtener el valor de los parámetros a variar. Se determina la velocidad del combustible a la salida del inyector para obtener el diámetro de salida y la longitud de este. También se determina la velocidad del combustible en el mezclador para obtener su geometría. Por último, se obtiene la potencia entregada por los combustibles y la eficiencia del quemador.

Esta investigación tiene como objetivo mejorar la eficiencia térmica de la cocina industrial. Además, se desea que los riesgos de operación sean mínimos en el manejo de la cocina industrial por los usuarios, se proyecta a trabajar en baja presión $2.8 \mathrm{kPa}$ ( $28 \mathrm{mbar}$ ) ya que en la actualidad se trabaja con la válvula del tanque de GLP completamente abierta, el cual almacena el gas a altas presiones $(600 \mathrm{kPa}$ manométricos aproximadamente). Además, se obtendrá un beneficio económico para los usuarios, ya que al mejorar la combustión se utilizará menor cantidad de combustible. Indirectamente se obtendrá un beneficio ambiental y en salud, ya que con una mejora de la eficiencia se tendrá una menor cantidad de emisiones de COx. 


\section{MATERIALES Y MÉTODOS}

Se realiza una descripción detallada de las ecuaciones, equipos e instrumentos y luego se presenta la metodología para determinar la eficiencia de la cocina industrial.

Diseño del mezclador

Se diseñó el quemador para que trabaje a una potencia de $3.75 \mathrm{~kW}$. Además, se tomará en cuenta las recomendaciones dadas por Fulford (Fulford, 1996).

Primero se realizará el análisis de la mezcla estequiométrica de aire - gas licuado de petróleo (GLP):

EI GLP utilizado está constituido en $55 \%$ por propano y $45 \%$ de butano, donde la mezcla estequiométrica es la siguiente:

$$
\left(0.55 \mathrm{C}_{3} \mathrm{H}_{8}+0.45 \mathrm{C}_{4} \mathrm{H}_{10}\right)+5.675\left(\mathrm{O}_{2}+3.76 \mathrm{~N}_{2}\right) \rightarrow 3.45 \mathrm{CO}_{2}+4.45 \mathrm{H}_{2} \mathrm{O}+21.338 \mathrm{~N}_{2}
$$

Haciendo uso de un exceso de aire de $10 \%$ con la ecuación (2) se obtiene el balance molar:

$$
\left(0.55 \mathrm{C}_{3} \mathrm{H}_{8}+0.45 \mathrm{C}_{4} \mathrm{H}_{10}\right)+6.2425\left(\mathrm{O}_{2}+3.76 \mathrm{~N}_{2}\right) \rightarrow 3.45 \mathrm{CO}_{2}+4.45 \mathrm{H}_{2} \mathrm{O}+23.4718 \mathrm{~N}_{2}+0.56 \mathrm{O}_{2}
$$

La relación de aire-gas combustible se calcula usando la ecuación (3) (Fulford, 1996):

$$
\mathrm{RAC}_{\mathrm{v}}=\frac{6.2425 \times 4.76 \times 24.8}{1 \times 24.8}=29.714
$$

La cantidad de aire primario que será utilizado en la mezcla será de $50 \%$ del total de aire requerido, tal como recomienda Fulford (1996). Esta recomendación se tendrá en cuenta para este caso. Es así que la relación de arrastre estará definida por:

$$
\mathrm{r}_{a r}=0.5 \times \mathrm{RAC}_{\mathrm{v}}
$$

Se obtiene, Para gas licuado de petróleo: $\mathrm{r}_{\mathrm{ar}}=14.857$

El diámetro de la garganta del mezclador tipo Venturi (Dt) se determinará mediante la fórmula de Prigg (Nasr, 2014):

$$
D_{t}=\left(\frac{\mathrm{r}_{a r}}{\sqrt{s}}+1\right) \times d_{i}
$$

Donde, $\mathrm{D}_{\mathrm{t}}=$ Diámetro de la garganta del mezclador $(\mathrm{m}) ; \mathrm{s}=$ Densidad relativa del gas combustible; $\mathrm{rar}_{\mathrm{r}}$ Relación de arrastre; di=Diámetro del inyector seleccionado $(\mathrm{m})$.

Usando la recomendación de Fulford (1996) se determina la longitud del venturi usando la ecuación (6):

$$
L_{t}=6 \times D_{t}
$$

Para determinar la geometría del difusor se toma en cuenta que el difusor debe tener un ángulo entre $5^{\circ}-8^{\circ}$ con respecto al eje central (Nasr, 2014):

$$
L_{d}=12 \times D_{t}
$$

Se obtiene, para gas licuado de petróleo: $\mathrm{Dt}=0.0125 \mathrm{~m}, \mathrm{Lt}=0.0749 \mathrm{~m}, \mathrm{Ld}=0.1498 \mathrm{~m}$

Luego de seguir las recomendaciones mencionadas se obtuvo lo siguiente: Para gas licuado de petróleo (GLP), el modelo cuenta con un diámetro de garganta de $0.01248 \mathrm{~m}$, el largo de la sección de entrada del mezclador denominado Venturi cuenta con una longitud de $0.07491 \mathrm{~m}$, mientras que la sección divergente denominada difusor cuenta con un largo de $0.1498 \mathrm{~m}$. Se utilizará un ángulo de $7^{\circ}$ con respecto al eje central para el diseño de la sección denominada Venturi, mientras que se utilizará un ángulo de $5^{\circ}$ con respecto al eje central para el diseño de la sección denominada difusor (Figura 1). 


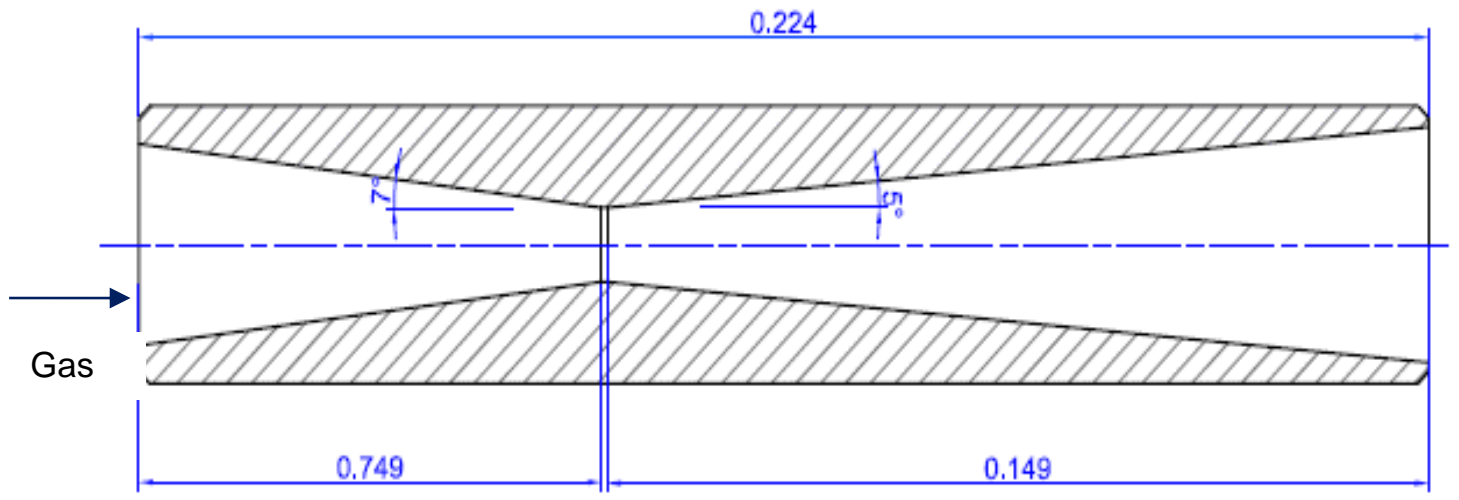

Fig. 1: Esquema del dimensionamiento del mezclador, (m)

Diseño de la cabeza del quemador

Siguiendo la recomendación de Nasr (2014) para la ecuación (5), el cual deriva de la fórmula de Prigg, se toma en cuenta las perdidas por fricción y el coeficiente de descarga. El diámetro resultante se toma como si solo hubiera un puerto para la salida de la llama (Nasr, 2014):

$$
D_{p}=\frac{D_{t}}{\sqrt{0.7}}
$$

Donde, $D_{p}=$ Diámetro de puerto de la llama $(m)$.

Se halla el área total del puerto de la llama, considerando como si fuera un solo agujero.

$$
A_{p}=\frac{\pi \times D_{p}^{2}}{4}
$$

Donde, $A_{p}=$ Área del puerto de la llama $\left(\mathrm{m}^{2}\right)$.

Para calcular la cantidad de agujeros se propondrá que el diámetro de puerto del agujero tenga un valor de $0.0015 \mathrm{~m}$

$$
N_{p}=\frac{4 \times A_{p}}{\pi \times d_{p}^{2}}
$$

Donde, $d_{p}=$ Diámetro de puerto de cabeza del quemador $(m) ; N_{p}=$ Numero de puertos en la cabeza del quemador.

Se obtiene, para gas licuado de petróleo: $D_{p}=0.01492 \mathrm{~m}, A_{p}=174.88 \times 10^{-6} \mathrm{~m}^{2}, \mathrm{~N}_{p}=99$ puertos

El quemador desarrollado debe ser sometido a ensayos variando distintos parámetros para poder determinar la eficiencia y evaluar la calidad de la mezcla aire-gas combustible generada en el proceso de combustión. Luego, se podrá determinar a qué parámetros se obtiene la mejor eficiencia y comparar cual fue el efecto al modificar la geometría del quemador.

\section{Análisis de Rendimiento Térmico}

Para determinar la eficiencia térmica de la cocina industrial se emplea dos normas técnicas INN-CHILE 2001 (INN-CHILE, 2001) y la GB 16410-2006 (GB National Standard of the People's Republic of China, 2006). Se procede a analizar y calcular el intercambio de energía que ocurre al calentar el agua hasta cierta temperatura según norma técnica utilizando el banco de ensayos del laboratorio de combustión y emisiones de la sección de Ingeniería Mecánica de la PUCP (Pontificia Universidad Católica del Perú). La eficiencia térmica es el cociente entre la energía absorbida por el $\mathrm{H}_{2} \mathrm{O}$ al calentarse hasta una temperatura dada por la norma técnica y la energía entregada por el combustible, y se formula de la siguiente manera:

$$
\eta=\frac{E_{\text {agua }}}{E_{\text {gas }}} \times 100 \%
$$

Donde, $\eta=$ eficiencia Térmica (\%); Eagua=Energía consumida por el agua (kJ); Egas=Energía entregada por el gas combustible (kJ). 
La energía absorbida por el agua se calcula mediante la ecuación (12). Se considera una masa de agua constante según el tamaño de olla a utilizar y según lo propone la norma técnica (INN-CHILE, 2001):

$$
\mathrm{E}_{\text {agua }}=\mathrm{m}_{\text {agua }} \cdot \mathrm{C}_{\text {agua }}\left(\mathrm{T}_{\mathrm{f}}-\mathrm{T}_{\mathrm{i}}\right)
$$

Donde, $\mathrm{C}_{\text {agua }}=$ Calor Específico de agua $\left(\mathrm{kJ} / \mathrm{kg} \cdot{ }^{\circ} \mathrm{C}\right) ; \mathrm{T}_{\mathrm{f}}=$ Temperatura Final del agua $\left({ }^{\circ} \mathrm{C}\right) ; \mathrm{T}_{\mathrm{i}}=$ Temperatura Inicial del agua $\left({ }^{\circ} \mathrm{C}\right)$.

Se realiza la medición del caudal de gas que es proporcionada a la cocina prototipo. Luego, se determina el tiempo de duración de calentamiento que alcanza el agua para llegar a la temperatura final requerida según norma técnica (INN-CHILE, 2001). Finalmente se emplea la ecuación (13) para determinar la energía suministrada por el gas combustible.

$$
\mathrm{E}_{\mathrm{gas}}=\dot{V}_{g} \cdot t_{a} \cdot P C I
$$

Donde, $\dot{\mathrm{V}}_{\mathrm{g}}=$ Flujo volumétrico del gas combustible $\left(\mathrm{m}^{3} / \mathrm{s}\right) ; t_{\mathrm{a}}=$ Tiempo requerido para calentar el agua (s); $\mathrm{PCl}=$ Poder Calorífico Inferior del gas combustible $\left(\mathrm{kJ} / \mathrm{m}^{3}\right)$.

Los ensayos a realizar serán siguiendo los pasos establecidos por la norma NCh927/1, elaborada por la División de Normas del Instituto Nacional de Normalización en Chile.

La eficiencia térmica se obtiene mediante la ecuación (14), (INN-CHILE 2001, 48):

$$
\eta=m_{a} * C_{\text {agua }} * \frac{T_{f}-T_{i}}{V * P C I} * 100
$$

Donde, $\mathrm{\eta}=$ eficiencia térmica, \%; $\mathrm{m}_{\mathrm{a}}=$ masa de agua, $\mathrm{kg} ; \mathrm{C}_{\text {agua }}=$ calor especifico del agua, $4.186 \mathrm{~kJ} /\left(\mathrm{kg} .{ }^{\circ} \mathrm{C}\right)$; $\mathrm{T}_{i}=$ temperatura inicial del agua, ${ }^{\circ} \mathrm{C} ; \mathrm{T}_{\mathrm{f}}=$ temperatura final del agua después de la extinción, en ${ }^{\circ} \mathrm{C} ; \dot{\mathrm{V}}_{\mathrm{g}}=$ volumen o masa de gas consumido, $\mathrm{m}^{3}$ (referido a las condiciones de referencia del poder calorífico); $\mathrm{PCl}=$ poder calorífico inferior del gas combustible, $\left(\mathrm{kJ} / \mathrm{m}^{3}\right)$. Los valores indicados en la norma para la realización de los ensayos son los siguientes (Tabla 1):

Tabla 1: Valores de las variables a utilizar en los ensayos (INN-CHILE, 2001)

\begin{tabular}{|l|c|c|}
\hline Variable & Unidad & Valor \\
\hline Masa de agua & $\mathrm{Kg}$ & 11.4 \\
\hline Calor específico del agua & $\mathrm{kJ} / \mathrm{kg}{ }^{\circ} \mathrm{C}$ & 4.186 \\
\hline Temperatura inicial del agua & ${ }^{\circ} \mathrm{C}$ & 27 \\
\hline Temperatura final del agua & ${ }^{\circ} \mathrm{C}$ & 90 \\
\hline
\end{tabular}

Siguiendo las indicaciones de la norma NCh927/1, se debe realizar un precalentamiento al quemador utilizando un recipiente de aluminio cuyo diámetro sea de $0.220 \mathrm{~m}$ y contenga $3.7 \mathrm{~kg}$ de agua en su interior durante $600 \mathrm{~s}$ (10 minutos). Inmediatamente después se procederá a realizar el ensayo con un recipiente de aluminio de $0.320 \mathrm{~m}$ de diámetro con un contenido de $11.4 \mathrm{~kg}$ de agua (INN-CHILE, 2001).

Para determinar la eficiencia térmica de la cocina prototipo y las emisiones de los gases de combustión se tendrá en cuenta lo siguiente: el diámetro del inyector será de $1 \mathrm{~mm}(\mathrm{GLP})$ y las presiones de ensayo serán $2.8,3.5,4.0,5.0$, y $6.0 \mathrm{kPa}(28,35,40,50$ y $60 \mathrm{mbarg})$. Además, se variará la altura entre el quemador y la base de la olla (Figura 3). Se mantendrá fijo la distancia entre el inyector y la entrada del mezclador, considerando $0 \mathrm{~m}(0 \mathrm{~mm})$ la distancia cuando el inyector se encuentra al nivel de la entrada del mezclador. Se eligió esta distancia porque se observó que hubo un correcto arrastre de aire, por ende, se obtuvo una mejor combustión (Figura 4). La tabla 3 y la tabla 4 presentan los datos de las pruebas obtenidas en este experimento.

En la tabla 2 se muestran los instrumentos utilizados en los ensayos. Las variables ambientales para los ensayos fueron: Presión barométrica 999.27 mbar, temperatura $28^{\circ} \mathrm{C}+/-2{ }^{\circ} \mathrm{C}$, humedad relativa $61.4 \%$, temperatura del gas $26^{\circ} \mathrm{C}+/-2^{\circ} \mathrm{C}$. 
Tabla 2: Características de los equipos de medición

\begin{tabular}{|c|c|c|c|}
\hline Instrumento & Rango & Precisión & Condiciones \\
\hline Indicador de temperatura ambiental & -20 a $80^{\circ} \mathrm{C}$ & $\begin{array}{l}+/-0.3^{\circ} \mathrm{C} \\
+/-0.4^{\circ} \mathrm{C}\end{array}$ & $\begin{array}{l}\text { De } 0 \text { a } 70^{\circ} \mathrm{C} \\
\text { Restante }\end{array}$ \\
\hline Indicador de humedad & 0 a 100 & $\begin{array}{l}+/-2.0 \% \\
+/-2.5 \% \\
\end{array}$ & $\begin{array}{c}\text { De } 10 \text { a } 90 \% \mathrm{RH} \\
\text { Restante }\end{array}$ \\
\hline Indicador de presión atmosférica & 600 a 1100 mbar & +/- 0.5 mbar @25² & \\
\hline Termocupla tipo $\mathrm{K}$ & 0 a $500{ }^{\circ} \mathrm{C}$ & Norma IEC584-Clase 2 & \\
\hline Medidor de flujo & 0 a 50 SLPM & $\begin{array}{c}+/-0.8 \% \text { de la lectura } \\
+/-0.2 \% \text { full }\end{array}$ & $\begin{array}{l}\text { Max presión de línea: } 145 \\
\text { psi Temperatura de } \\
\text { operación: }-10 \text { a } 50^{\circ} \mathrm{C}\end{array}$ \\
\hline Manómetro digital & -1 a 2 bar & $0.05 \% F E$ & $\begin{array}{c}\text { Resolución: } 0.1 \text { mbar } \\
\text { Temperatura de trabajo: } 0 \\
\text { a } 50^{\circ} \mathrm{C}\end{array}$ \\
\hline Analizador de gases (Quintox) & & & Incertidumbre: +/- 20 ppm \\
\hline
\end{tabular}

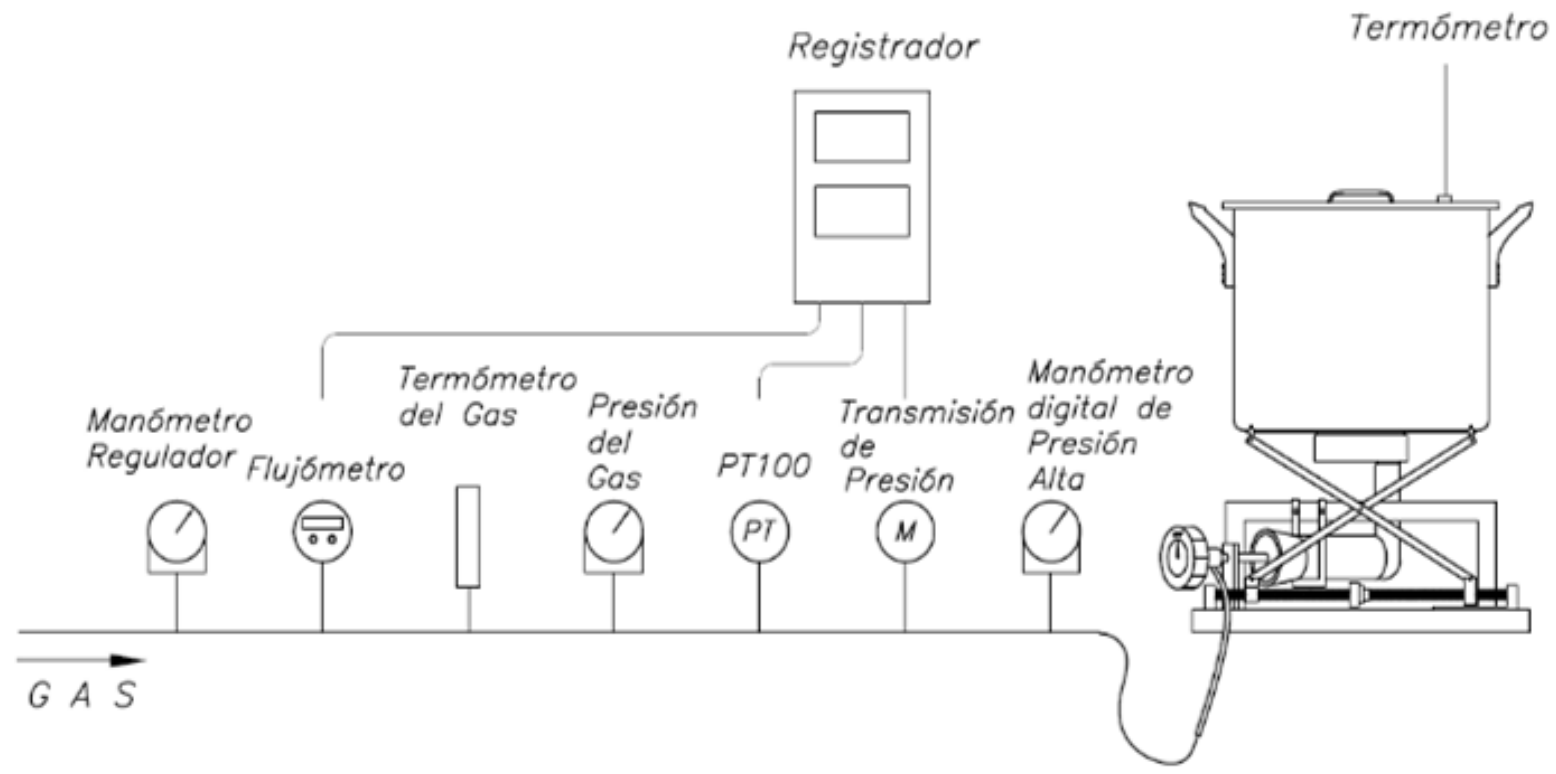

Fig. 2: Esquema del banco de ensayos

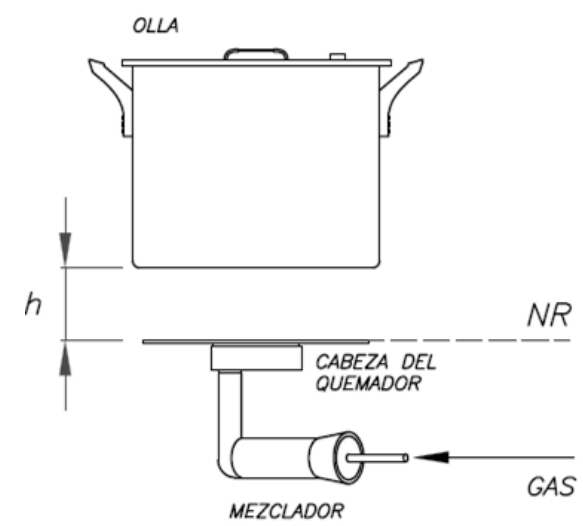

Fig. 3: Altura (h) entre la base de la olla y la cabeza del quemador

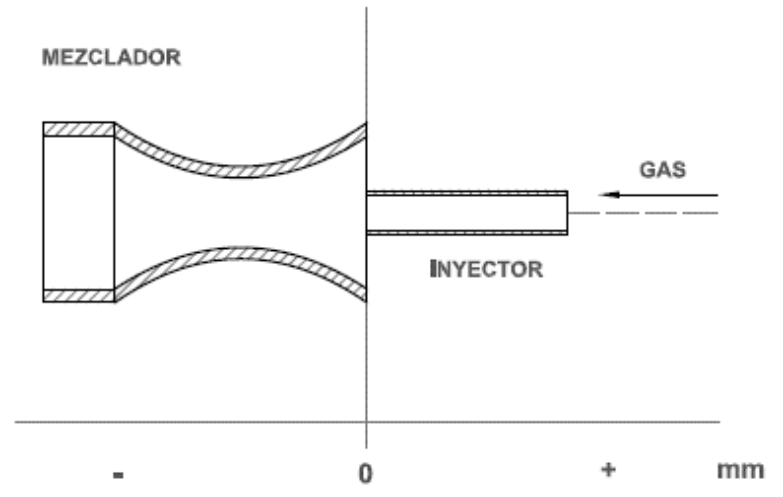

Fig. 4: Esquema de la entrada del mezclador - inyector

\section{RESULTADOS Y DISCUSIÓN}

A continuación, se muestran los resultados de los diferentes ensayos realizados en un banco de pruebas. El término $\eta^{*}$ es la eficiencia térmica según la norma técnica GB 16410-2006. 
Tabla 3: Datos y resultados variando la altura entre la base de la olla y el quemador

\begin{tabular}{|c|c|c|c|c|c|c|c|c|c|c|}
\hline Ensayo & $\mathrm{di}(\mathrm{m})$ & $\mathrm{h}(\mathrm{m})$ & $\mathrm{Pg}(\mathrm{kPa})$ & $\mathrm{V}(\mathrm{LPM})$ & $\mathrm{P}(\mathrm{kW})$ & $\mathrm{Ti}\left({ }^{\circ} \mathrm{C}\right)$ & $\mathrm{T}_{\mathrm{f}}\left({ }^{\circ} \mathrm{C}\right)$ & $\mathrm{t}(\mathrm{s})$ & $\eta(\%)$ & $\eta^{*}(\%)$ \\
\hline 1 & 0.001 & 0.020 & 2.81 & 1.99 & 3.170 & 30 & 93 & 1714 & 55.32 & 57.38 \\
\hline 2 & 0.001 & 0.025 & 2.83 & 1.99 & 3.170 & 30 & 93 & 1660 & 57.12 & 59.73 \\
\hline 3 & 0.001 & 0.030 & 2.80 & 1.97 & 3.139 & 30 & 93 & 1758 & 54.49 & 56.59 \\
\hline 4 & 0.001 & 0.035 & 2.82 & 1.98 & 3.155 & 30 & 93 & 1820 & 52.36 & 54.81 \\
\hline
\end{tabular}

Tabla 4: Datos y resultados variando la presión del gas licuado de petróleo

\begin{tabular}{|c|c|c|c|c|c|c|c|c|c|c|}
\hline Ensayo & $\mathrm{di}(\mathrm{m})$ & $\mathrm{h}(\mathrm{m})$ & $\mathrm{Pg}(\mathrm{kPa})$ & $\mathrm{V}(\mathrm{LPM})$ & $\mathrm{P}(\mathrm{kW})$ & $\mathrm{Ti}\left({ }^{\circ} \mathrm{C}\right)$ & $\mathrm{T}_{\mathrm{f}}\left({ }^{\circ} \mathrm{C}\right)$ & $\mathrm{t}(\mathrm{s})$ & $\eta(\%)$ & $\begin{array}{c}\eta^{*} \\
(\%)\end{array}$ \\
\hline 1 & 0.001 & 0.025 & 6.01 & 2.93 & 4.668 & 30 & 93 & 1185 & 54.35 & 55.46 \\
\hline 2 & 0.001 & 0.025 & 5.00 & 2.68 & 4.270 & 30 & 93 & 1284 & 54.84 & 55.70 \\
\hline 3 & 0.001 & 0.025 & 4.00 & 2.40 & 3.824 & 30 & 93 & 1424 & 55.21 & 56.81 \\
\hline 4 & 0.001 & 0.025 & 3.50 & 2.22 & 3.537 & 30 & 93 & 1490 & 57.05 & 59.52 \\
\hline 5 & 0.001 & 0.025 & 2.83 & 1.99 & 3.170 & 30 & 93 & 1660 & 57.12 & 59.73 \\
\hline
\end{tabular}

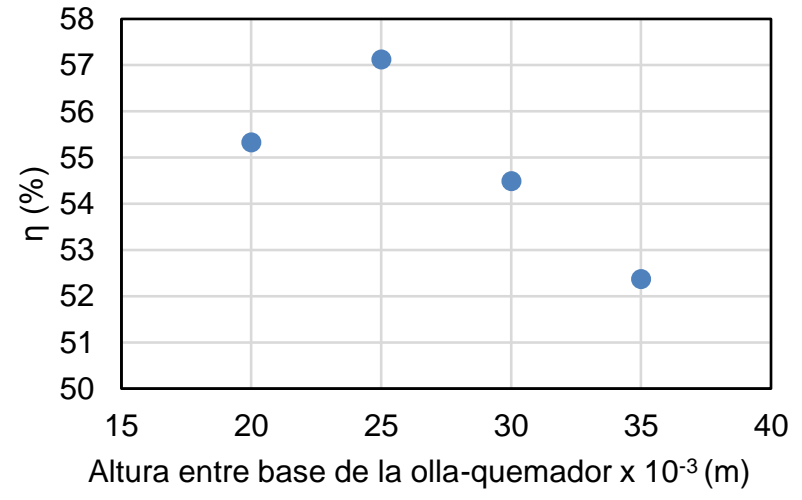

Fig. 5: Rendimiento vs. Altura entre la base de la olla y quemador

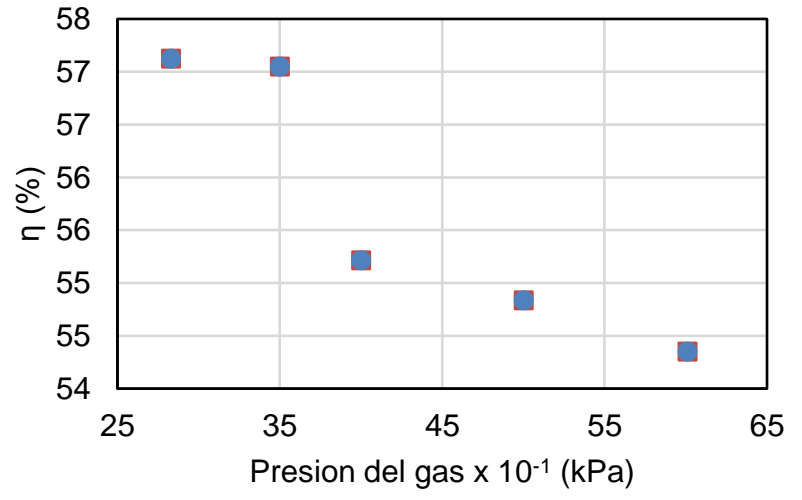

Fig. 6: Rendimiento vs. Presión manométrica del gas

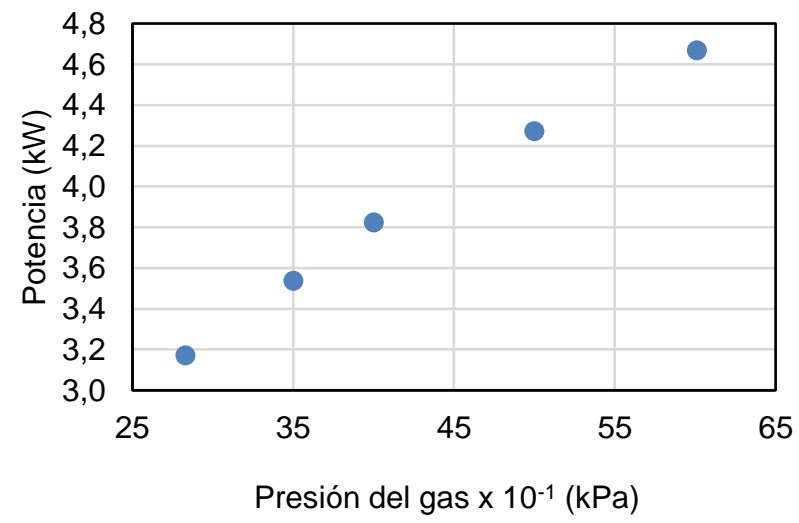

Fig. 7: Potencia entregada por el gas vs. Presión del gas

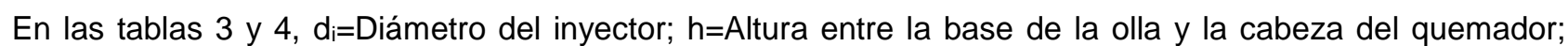
$\mathrm{P}_{\mathrm{g}}=$ Presión manométrica del gas combustible; $\dot{\mathrm{V}}_{\mathrm{g}}=$ Flujo volumétrico del gas combustible; $\mathrm{P}=$ Potencia térmica; $\mathrm{t}=$ Tiempo de calentamiento del agua (equivalente a $\mathrm{t}_{\mathrm{a}}$ ). En la tabla 3 se puede observar que el punto de mayor rendimiento térmico fue cuando la distancia entre la base de la olla y el quemador fue de $0.025 \mathrm{~m}$. A medida que se incrementó dicha distancia $0.005 \mathrm{~m}(5 \mathrm{~mm})$, se obtuvo que la eficiencia fue bajando entre $2 \%$ y $2.5 \%$ aproximadamente. Cuando la distancia entre la base de la olla y el quemador fue menor a $0.025 \mathrm{~m}$, la eficiencia bajo $1.8 \%$. Además, se observó que la llama no pudo desarrollarse completamente, se mostró inestable y se produjo una mayor cantidad de hollín.

En la Figura 6 se puede observar que a medida que la presión del gas fue disminuyendo desde 6.0 kPa a 2.8 $\mathrm{kPa}$, el rendimiento térmico fue mejorando. Se obtuvo un rendimiento de $54.4 \%$ cuando se trabajó con una 
presión de $6.0 \mathrm{kPa}$ aproximadamente, mientras que se obtuvo un rendimiento de $57.1 \%$ cuando se trabajó con una presión de $2.8 \mathrm{kPa}$ aproximadamente, que es la presión con la que debe trabajar el quemador cuando se utiliza GLP como combustible. El quemador diseñado puede operar en un rango de potencias de $3-4.6$ $\mathrm{kW}$ tal como se ve en la Figura 7, estas potencias pueden ser reguladas al cambiar la presión del gas a la que opera el quemador. Además, luego de $4.6 \mathrm{~kW}$ se produce desprendimiento de llama, lo cual lo hace peligroso para el usuario. Las Tablas 5, 6, 7 y 8 presentan los datos obtenidos en el ensayo de emisiones utilizando como combustible gas licuado de petróleo (GLP), visualizándose que las concentraciones de $\mathrm{NO}_{2}, \mathrm{NO} \times, \mathrm{SO}_{2}$, $\mathrm{CO}$ y $\mathrm{C} \times \mathrm{H}$ y aumentan a medida que se utiliza mayor presión y flujo de gas. Las Figuras 8 y 9 muestran el comportamiento del $\mathrm{SO}_{2}$ y $\mathrm{CO}$ cuando la presión del gas es de $2.8 \mathrm{kPa}$ aproximadamente. Se puede comparar las emisiones a diferentes alturas con una misma presión $(2.8 \mathrm{kPa})$, por lo que se puede concluir que la menor concentración de $\mathrm{SO}_{2}$ y $\mathrm{CO}$ se obtuvo cuando la altura entre la base de la olla y quemador fue de $0.020 \mathrm{~m}$.

Tabla 5: Datos de emisiones a una altura de $0.020 \mathrm{~m}$ entre la base de la olla y cabeza del quemador

\begin{tabular}{|c|c|c|c|c|c|c|c|c|}
\hline $\mathrm{V}(\mathrm{LPM})$ & $\mathrm{Pg}(\mathrm{kPa})$ & $\mathrm{NO}(\mathrm{ppm})$ & $\mathrm{NO}_{2}(\mathrm{ppm})$ & $\mathrm{NOx}(\mathrm{ppm})$ & $\mathrm{SO}_{2}(\mathrm{ppm})$ & $\mathrm{CO}(\mathrm{ppm})$ & $\mathrm{CO}_{2 \mathrm{~m}}(\%)$ & $\mathrm{C}_{\mathrm{X}} \mathrm{H}_{\mathrm{Y}}(\mathrm{ppm})$ \\
\hline 1.96 & 2.80 & 2 & 14 & 16 & 274 & 1267 & 5.7 & 67 \\
\hline 2.21 & 3.52 & 3 & 16 & 19 & 312 & 1426 & 6.6 & 54 \\
\hline 2.37 & 4.01 & 3 & 17 & 20 & 594 & 1930 & 7.1 & 85 \\
\hline 2.66 & 5.01 & 3 & 17 & 20 & 503 & 2313 & 7.1 & 68 \\
\hline 2.93 & 6.01 & 3 & 15 & 18 & 573 & 2522 & 7.5 & 94 \\
\hline
\end{tabular}

Tabla 6: Datos de emisiones a una altura de $0.025 \mathrm{~m}$ entre la base de la olla y cabeza del quemador

\begin{tabular}{|c|c|c|c|c|c|c|c|c|}
\hline $\mathrm{V}(\mathrm{LPM})$ & $\mathrm{Pg}(\mathrm{kPa})$ & $\mathrm{NO}(\mathrm{ppm})$ & $\mathrm{NO}_{2}(\mathrm{ppm})$ & $\mathrm{NOx}(\mathrm{ppm})$ & $\mathrm{SO}_{2}(\mathrm{ppm})$ & $\mathrm{CO}(\mathrm{ppm})$ & $\mathrm{CO}_{2 \mathrm{~m}}(\%)$ & $\mathrm{C}_{\mathrm{X}} \mathrm{H}_{\mathrm{Y}}(\mathrm{ppm})$ \\
\hline 1.96 & 2.80 & 1 & 11 & 12 & 298 & 1478 & 5.6 & 90 \\
\hline 2.21 & 3.52 & 1 & 16 & 17 & 403 & 1956 & 6.9 & 104 \\
\hline 2.37 & 4.01 & 1 & 16 & 17 & 493 & 2585 & 7.0 & 129 \\
\hline 2.66 & 5.01 & 3 & 16 & 19 & 717 & 3652 & 7.7 & 175 \\
\hline 2.93 & 6.01 & 3 & 18 & 21 & 887 & 4079 & 7.7 & 161 \\
\hline
\end{tabular}

Tabla 7: Datos de emisiones a una altura de $0.030 \mathrm{~m}$ entre la base de la olla y cabeza del quemador

\begin{tabular}{|c|c|c|c|c|c|c|c|c|}
\hline $\mathrm{V}(\mathrm{LPM})$ & $\mathrm{Pg}(\mathrm{kPa})$ & $\mathrm{NO}(\mathrm{ppm})$ & $\mathrm{NO}_{2}(\mathrm{ppm})$ & $\mathrm{NOx}(\mathrm{ppm})$ & $\mathrm{SO}_{2}(\mathrm{ppm})$ & $\mathrm{CO}(\mathrm{ppm})$ & $\mathrm{CO}_{2 \mathrm{~m}}(\%)$ & $\mathrm{C}_{x} \mathrm{Hr}_{\mathrm{r}}(\mathrm{ppm})$ \\
\hline 1.96 & 2.80 & 2 & 12 & 14 & 346 & 1754 & 5.6 & 67 \\
\hline 2.21 & 3.52 & 2 & 14 & 16 & 373 & 1904 & 6.1 & 61 \\
\hline 2.37 & 4.01 & 2 & 15 & 17 & 331 & 1573 & 6.5 & 47 \\
\hline 2.66 & 5.01 & 1 & 15 & 16 & 488 & 2360 & 7.3 & 86 \\
\hline 2.93 & 6.01 & 1 & 13 & 14 & 841 & 4088 & 7.2 & 176 \\
\hline
\end{tabular}

Tabla 8: Datos de emisiones a una altura de $0.035 \mathrm{~m}$ entre la base de la olla y cabeza del quemador

\begin{tabular}{|c|c|c|c|c|c|c|c|c|}
\hline $\mathrm{V}(\mathrm{LPM})$ & $\mathrm{Pg}(\mathrm{kPa})$ & $\mathrm{NO}(\mathrm{ppm})$ & $\mathrm{NO}_{2}(\mathrm{ppm})$ & $\mathrm{NOx}(\mathrm{ppm})$ & $\mathrm{SO}_{2}(\mathrm{ppm})$ & $\mathrm{CO}(\mathrm{ppm})$ & $\mathrm{CO}_{2 \mathrm{~m}}(\%)$ & $\mathrm{C}_{\mathrm{X}} \mathrm{H}_{\mathrm{Y}}(\mathrm{ppm})$ \\
\hline 1.96 & 2.80 & 1 & 7 & 8 & 315 & 2050 & 5.7 & 68 \\
\hline 2.21 & 3.52 & 1 & 14 & 15 & 298 & 1928 & 6.5 & 63 \\
\hline 2.37 & 4.01 & 1 & 16 & 17 & 505 & 2475 & 6.7 & 85 \\
\hline 2.66 & 5.01 & 1 & 17 & 18 & 521 & 2891 & 7.6 & 97 \\
\hline 2.93 & 6.01 & 3 & 17 & 20 & 741 & 3679 & 7.4 & 136 \\
\hline
\end{tabular}

De los resultados obtenidos se discute que hay concordancia en el aumento de eficiencia térmica cuando se toma en cuenta el diseño apropiado del quemador según lo menciona Aroojattham y Suttikiat (2015); hay concordancia que aumenta la eficiencia térmica cuando se toma en cuenta la distancia entre el recipiente y el quemador según Agarwal et al. (2015); hay concordancia con lo que manifiesta Surange (2014) que el desempeño de la cocina se afecta con el diseño del quemador; hay concordancia con Del (2009) al manifestar que hay un mejor desempeño de la cocina al considerar las secciones de convergencia en el mezclador; también con otros autores como Agudelo y Salinas (2010), Cancino et al. (2007), y Mishra et al. (2015) que manifiestan mejor desempeño de la cocina teniendo en cuenta el diseño del quemador de premezcla. 


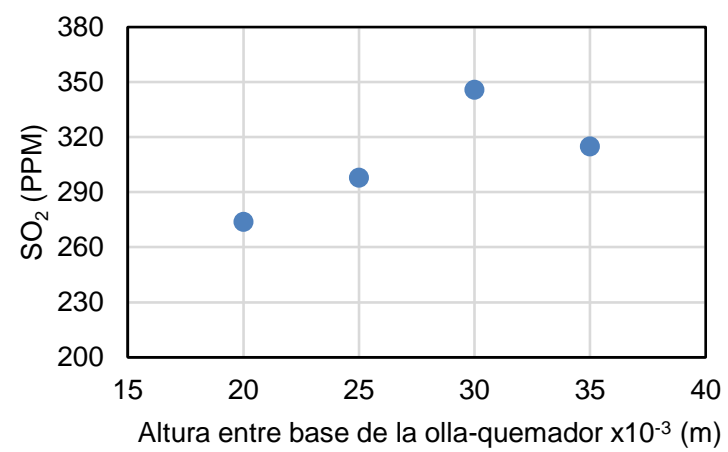

Fig. 8: Dióxido de azufre $\left(\mathrm{SO}_{2}\right)$ vs. Altura entre la base de la olla y quemador

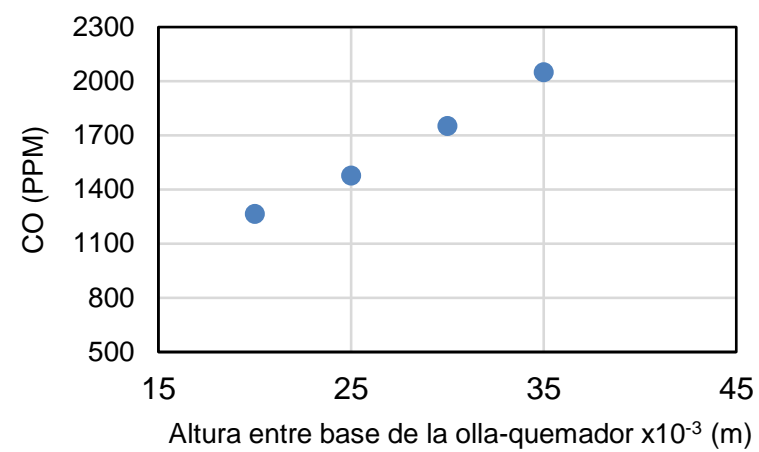

Fig. 9: Monóxido de carbono vs. Altura entre la base de la olla y quemador

\section{CONCLUSIONES}

Se concluye que la mayor eficiencia térmica fue cuando la distancia entre la base de la olla y el quemador estaba a $0.025 \mathrm{~m}$, y por debajo de esta medida se observó que la llama no pudo desarrollarse completamente, se mostró inestable y se produjo una mayor cantidad de hollín. Además, cuando la presión del gas fue disminuyendo desde $6.0 \mathrm{kPa}$ a $2.8 \mathrm{kPa}$, la eficiencia térmica fue mejorando. Se pudo constatar que la eficiencia térmica obtenida mediante la norma técnica latinoamericana es entre 1 a $2 \%$ menor a la obtenida mediante la norma técnica asiática. Se constató que la menor concentración de emisiones se dio cuando se utilizó una presión de gas de $2.8 \mathrm{kPa}$. Finalmente se determina que la menor concentración de $\mathrm{SO}_{2}$ y $\mathrm{CO}$ se obtuvo cuando la altura entre la base de la olla y quemador fue de $0.020 \mathrm{~m}$.

\section{AGRADECIMIENTOS}

Se agradece a Dirección de Gestión de la Investigación (DGI) que a través de los Proyectos Concursables de Investigación Básica y Aplicada 465-DGI-2017 de la Pontificia Universidad Católica del Perú que financiaron la investigación.

\section{REFERENCIAS}

Agarwal, P., A. Anand y R. Gupta, Performance Analysis of Conventional Lpg Cooking Stove, doi: 10.18000 , International Journal on Applied Bioengineering, 9(January), 15-19 (2015)

Agencia Internacional de la Energía, Consumo de energía procedente de combustibles fósiles (2014)

Agudelo, J., y W. Salinas, Estudio Teorico - Experimental de Quemadores de Premezcla con Aire Inductor, Para su Aplicación a la Pequena y Mediana Industria en Colombia, 219 (2010)

Aroonjattham, P., y S. Suttikiat, The Effects of High Pressure Gas Burner Parameters on Thermal Efficiency for 2014 International Conference on Machining, Materials and Mechanical Technology (IC3MT ), 657, 729-734 (2015)

Basu, D., R. Saha, R. Ganguly y A. Datta, Performace improvement of LPG cook stoves through burner and nozzle modifications, doi: 014426008X370951, Journal of the Energy Institute, 81(4), 218-225 (2008)

Cancino, L. R., A.H. Restrepo y A.A. Oliveira, Análisis numérico de la combustión en un quemador atmosférico tipo corona de aplicación doméstica, doi: 0122-1701, Scientia Et Technica, (35), 201-206 (2007)

Cengel, Y. a. y M.E. Boles, Termodinamica - Cengel $7^{\text {th }}$, Termodinamica, 1456 (2011)

Del, M., X.V. Congreso, I. Anual y D.E. Somim, Análisis numérico del flujo en un mezclador de aire y combustible con sección variable, 976-982 (2009)

Fooladgar, E. y C.K. Chan, Effects of stratification on flame structure and pollutants of a swirl stabilized premixed combustor, doi: 10.1016/j.applthermaleng.2017.05.197, Applied Thermal Engineering, 124 (2017)

Fulford, D., Biogas Stove Design. Biomass, (August), 1-21 (1996)

GB National Standard of the People's Republic of China, Domestic gas cooking appliances (2006)

INN-CHILE. Artefactos de uso doméstico para cocinar, que utilizan combustibles gaseosos - Parte 1: Requisitos generales de fabricación y métodos de ensayo (2001)

Mishra, N.K., S.C. Mishra y P. Muthukumar, Performance characterization of a medium-scale liquefied petroleum gas cooking stove with a two-layer porous radiant burner, doi:10.1016/j.applthermaleng.2015.05.077, Applied Thermal Engineering, 89 (2015) 
Nasr, G. y N.E. Connor, Natural Gas Engineering and Safety Challenges, Downstream Process, Analysis, Utilization and Safety, Springer, Cham, Online ISBN: 978-3-319-08948-5 (2014)

Ramos, B.G., Análisis experimental de cocinas anafe a gas licuado de petróleo y gas natural para alturas, Pontificia Universidad Católica del Perú, PUCP (2016)

Rojas, F.J., F. Jiménez, B. Ramos y R. Alván, Análisis Experimental del Rendimiento Térmico, Potencia y Emisiones de Cocinas Anafe a Gas Licuado de Petróleo para Altitudes entre 2200 Y 4200 metros, doi: 10.4067/S071807642017000400020, Información Tecnológica, 28(4), 179-190 (2017)

Rojas, F. J., F. Jiménez y J. Álvarez, Diseño y Evaluación de Mezcladores de un Quemador Doméstico usando Gas Licuado de Petróleo, doi: 10.4067/S0718-07642017000500019, Información Tecnológica, 28(5), 191-214 (2017)

SENAMHI. Evaluación de la calidad del aire en Lima Metropolitana 2014, Lima (2014)

Serrano, J., e Y. Carranza, Analisis teórico de la combustión en quemadores de gas natural. Scientia Et Technica, (29), 139-143 (2005)

Surange, J. R., N.K. Patil y A.V. Rajput, International Journal of Innovative Research in Science, Engineering and Technology Performance Analysis of Burners used in LPG Cooking Stove-A Review, 3(4), 87-97 (2014) 